\title{
A Rare Case of Aeroportia Due to Acute Gastric Dilatation - when the Radiologist Goes beyond the Imagess
}

\author{
Claudia-Gabriela Moldovanu' ${ }^{1}$, Cosmin Caraiani ${ }^{2,3}$, Bogdan Furnea ${ }^{3}$, Florin Graur ${ }^{2,3}$
}

1) Emergency Clinical County Hospital Cluj-Napoca; University of Medicine, Pharmacy, Science and Technology of Târgu Mureş; 2) Iuliu Hatieganu University of Medicine and Pharmacy, Cluj-Napoca; 3) Regional Institute of Gastroenterology and Hepatology Cluj-Napoca, Romania
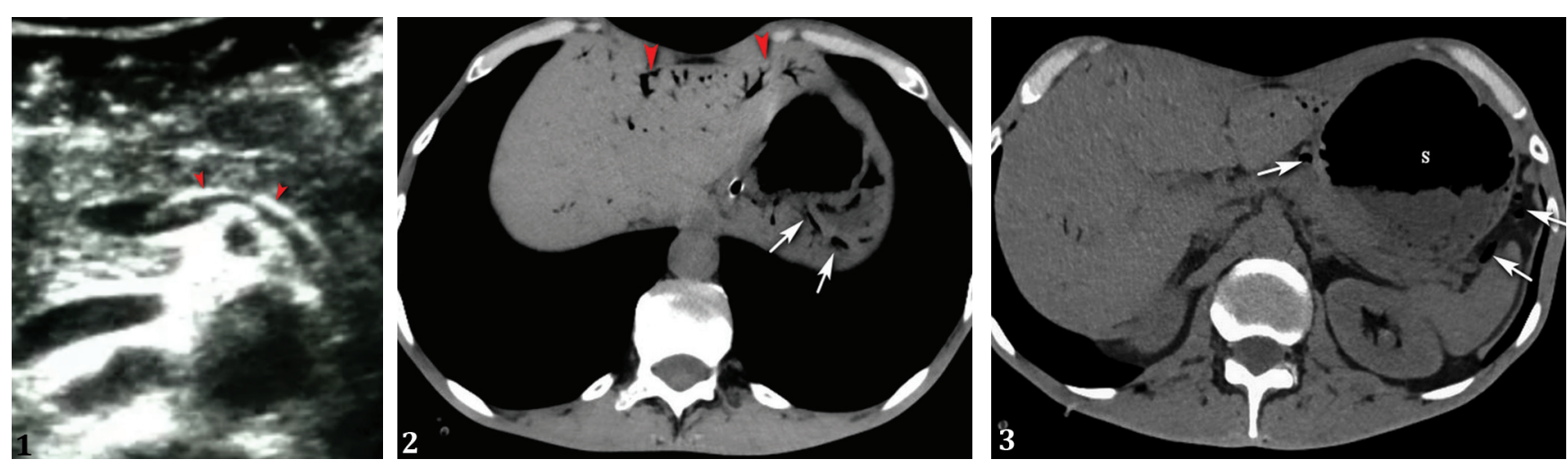

A 43-year-old male presented with a 3-week history of severe, diffuse abdominal pain associated with postprandial vomiting. He was previously diagnosed with peptic ulcer. The emergency abdominal ultrasonography detected the presence of gas in the splenic vein, seen as a linear, hyperechoic stripe in the anterior, non-dependent part of the vein wall (Fig. 1, red arrows). Due to increased serum creatinine level, an unenhanced computed tomography (CT) scan of the abdomen was performed, which revealed extensive portal venous gas (Fig. 2, red arrows) and a distended stomach, with gas localized next to the stomach wall (Figs. 2 and 3, white arrows). In the absence of imaging signs of mesenteric infarction, the radiologist suggested the most likely etiology for aeroportia, the presence of gastric outlet obstruction. The patient underwent upper digestive endoscopy, which revealed two deep ulcers, one in the gastric antrum and another in the anterior wall of the duodenal bulb, associated with an inflammatory stenosis that cannot be overcome with the endoscope. A HoffmeisterFinsterer gastrectomy was performed. The evolution was favorable.

Hepatic portal venous gas referred to as "aeroportia" is a radiological feature described when intraluminal gas or gas produced by gut bacteria enters the portal vein and its branches. The main etiology is intestinal ischemia, but other causes have been reported [1]. When it is caused by acute gastric dilatation, the exact pathogenic mechanism is still unknown; it might be due to increased intraluminal pressure. The high pressure might move the gas from the gastric mucosa to the venous system and lymphatics into the portal venous system $[1,2]$. It must be differentiated from pneumobilia, defined as the presence of gas in the biliary tree [3]. CT findings can help to differentiate them, the pneumobilia is centrally located and air extends to more than $2 \mathrm{~cm}$ from the liver capsule, whereas the aeroportia is located predominantly in the periphery [4].

Corresponding author: Cosmin Caraiani, ccaraiani@yahoo.com

Conflicts of interest: None to declare.

\section{REFERENCES}

1. Ortega-Deballon P, Radais F, Facy O. Aeroportia and acute abdominal pain: ischemic bowel necrosis. Clin J Gastroenterol 2008;1:157-159. doi:10.1007/s12328-008-0026-Z

2. Pereira M, Afonso LM, Patrao S, Araujo R. Aeroportia - A Rare Finding for a Frequent Disease. Clin Med Rev Case Rep 2016;3:089. doi:10.23937/2378-3656/1410089

3. Sherman SC, Tran H. Pneumobilia: benign or life-threatening. J Emerg Med 2006;30:147-153. doi:10.1016/j.jemermed.2005.05.016

4. Alqahtani S, Coffin CS, Burak K, Chen F, MacGregor J, Beck P. Hepatic portal venous gas: a report of two cases and a review of the epidemiology, pathogenesis, diagnosis and approach to management. Can J Gastroenterol 2007;21:309-313. doi:10.1155/2007/934908 